Психология. Журнал Высшей школы экономики,

2021. T. 18. № 3. C. 643-655. DOI: 10.17323/1813-8918-2021-3-643-655

\title{
ПСИХОЛОГИЧЕСКИЕ МЕТОДЫ СНИЖЕНИЯ ФУНКЦИОНАЛЬНОЙ ФИКСИРОВАННОСТИ
}

\author{
С.Р. ЯГОЛКОВСКИЙ
}

${ }^{a}$ Национальный исследовательский университет «Высшая школа экономики», 101000, Россия, Москва, ул. Мясницкая, д. 20

\begin{abstract}
Резюме
В настоящей статье представлено продолжение исследования феномена функциональной фиксированности, негативно влияющего на процесс творческого мышления и ограничивающего его эффективность. Описаны и кратко проанализированы основные психологические методы снижения функциональной фиксированности. Основной акцент в них делается на обогащении и расширении ассоциативного пространства, служащего источником идей и нестандартных решений в рамках творческой деятельности субъекта. Подчеркивается связь этих методов с теоретическими моделями и разработками, представленными в научно-психологической литературе (например: теорией «забывания фиксации», гипотезой «оппортунистической ассимиляции» и пр.). Некоторые из этих методов основываются на общих рекомендациях по ведению творческого процесса, другие же ориентированы на использование конкретных инструкций по его оптимизации. Описываются основные параметры инструкции, а также наиболее эффективные способы ее предъявления субъекту, осуществляющему творческую деятельность. Уделяется внимание той роли в снижении уровня функциональной фиксированности, которую выполняют процессы категоризации объектов, обращения субъекта к прошлому опыту, а также отвлечения субъекта от осуществляемой мыслительной деятельности. Кроме этого вкратце рассмотрены подходы, рассматривающие функциональную фиксированность не только как антагониста креативности, но и в некоторых случаях как фактор повышения эффективности творческого мышления. Это происходит в тех случаях, когда субъект отчетливо осознает влияние прошлого опыта на процесс решения творческой задачи и достаточно глубоко понимает ее структуру. Указывается на то, что в научных исследованиях функциональной фиксированности наметилась тенденция к выделению и анализу ее отдельных компонентов, что в ближайшем будущем может привести к появлению более таргетированных методов ее ослабления.
\end{abstract}

Ключевые слова: функциональная фиксированность, творческое мышление, установка, дивергентное мышление.

Функциональная фиксированность является негативным фактором, существенно ограничивающим продуктивность творческого мышления. В научной литературе представлен целый ряд классических (например: Adamson, 1952; Duncker, 1945; Kearsley, 1975) и современных (например: Munoz-Rubke et al., 2018) работ, которые направлены на исследование природы и сущности этого

Исследование выполнено при финансовой поддержке РФФИ, проект № 19-113-50017. 
феномена (ранее нами был сделан обзор и краткий анализ таких работ Медведев, Яголковский, 2020). Но при этом представлено также достаточно много разработок, ориентированных на поиск способов снятия или ослабления функциональной фиксированности. Анализу таких разработок, представленных в зарубежной научно-психологической литературе, посвящена настоящая статья.

Методы снижения функциональной фиксированности в рамках решения задач могут быть разделены по степени общности рекомендаций, которые они предлагают. Так, С. Олссон (Ohlsson, 1992) советует идти по пути обогащения или переработки уже имеющейся информации, связанной с задачей, однако подробных инструкций, как это сделать, не дает.

Более эффективными для ослабления функциональной фиксированности могут оказаться конкретные рекомендации. Так, например, она может быть ослаблена в рамках групповой творческой деятельности. В процессе обмена идеями участники группы могут предоставлять друг другу новую информацию о задаче и, таким образом, осуществлять взаимную стимуляцию креативности на когнитивном уровне (Coskun et al., 2000; Voiskounsky et al., 2017).

При формировании рабочей группы, реализующей творческую деятельность, необходимо уделить особое внимание созданию благоприятной и дружественной атмосферы. Так, в исследовании П.Дж. Карневейла и Т.М. Пробст (Carnevale, Probst, 1998) изучалось влияние конфликтных ситуаций или их ожидания в рамках решения творческих задач. Было продемонстрировано, что конфликтная ситуация или ее ожидание могут в значительной степени снизить общую способность к продуцированию творческих решений в силу возрастания роли ограничивающих творческое мышление факторов, в том числе и функциональной фиксированности.

Некоторые из авторов, рассматривавших проблему стимулирования творческого процесса, основываются на собственном представлении о его элементах, как стимулирующих, так и ограничивающих его продуктивность. Так, A. Маккафри (МсCaffrey, 2012) отмечает, что зачастую основным препятствием на пути решения творческой задачи является неспособность субъекта обратить внимание на некоторые скрытые и неявные особенности (obscure features) проблемы. Функциональная фиксированность в этой связи рассматривается как склонность субъекта упускать из внимания особенности предметов, материалов для решения задачи, вызванную прочной ассоциацией этого предмета или его частей с теми или иными конкретными функциями. В качестве «упускаемых» признаков предметов выделяются следующие: детали, материал, форма и размер. На основе этого предположения был предложен метод стимуляции творческого мышления generic-parts technique (GPT), в рамках которого субъекту перед началом поиска решения задачи предлагается обратить внимание на предложенные ему/ей предметы и распределить информацию о них по вышеназванным четырем признакам.

Ряд исследований выявил влияние формы инструкции, даваемой субъекту, на силу и выраженность возникающей у него/нее функциональной фиксированности (Chrysikou, Weisberg, 2005; Frank, Ramscar, 2003). Фактически опи- 
сывается возможность создания «дефиксирующих» инструкций. Такие инструкции могут специально акцентироваться на нежелательности использования в решении задачи некоторых конкретных «проблемных» элементов, представленных в примерах. Однако следует отметить, что инструкция сработает только в том случае, если субъект внимательно ее прочитает, поскольку само предъявление примера повышает вероятность невнимательного прочтения инструкции (Chi et al., 1981; LeFevre, Dixon, 1986; Pirolli, Anderson, 1985).

Е. Хрисикоу и др. (Chrysikou et al., 2016) предположили, что влияние на степень проявления функциональной фиксированности может оказывать не только содержание инструкции, но и то, в каких модальностях она представлена. В основу данного предположения было положено следующее наблюдение: изображение предмета в большей степени ассоциировано с некоторым опытом реального взаимодействия с ним, действиями, которые субъект совершал с этим предметом в прошлом; термин же, обозначающий этот предмет, в большей степени ассоциирован у субъекта с абстрактно-логической информацией об этом предмете (Glaser, Glaser, 1989; Saffran et al., 2003). Таким образом, стимулы различной модальности могут быть связаны с различными областями памяти о предмете. Результаты исследования продемонстрировали, что в рамках генерации добавочных функций предмета предъявление его изображения чаще будет приводить к активации нисходящей (top-down) стратегии семантического поиска, что приведет к усилению феномена функциональной фиксированности.

С. Глюксберг и Р. Вайсберг (Glucksberg, Weisberg, 1966) в своих исследованиях продемонстрировали, что называние всех элементов предложенной задачи способно устранить или сильно ослабить негативное воздействие функциональной фиксированности. Они предположили, что суть функциональной фиксированности заключается в потере субъектом возможности воспринимать функционально фиксированный объект как релевантный актуальной задаче. Развитие этой идеи можно найти в работе Глюксберга и Данкса (Glucksberg, Danks, 1967). В данной статье авторы рассуждают о возможной роли изучения феномена функциональной фиксированности в рамках формирования общего понимания процессов развития творческого мышления. Выход за рамки функциональной фиксированности предполагает нахождение некоторого нового способа использования предмета. А когда объект используется по-новому, он может функционально оказаться эквивалентным тому объекту, который необходим для решения творческой задачи. Глюксберг и Данкс приводят следующий пример: решение «проблемы цепи» требует, чтобы испытуемый использовал металлический инструмент в качестве замены провода для замыкания электрической цепи. Таким образом, металлический инструмент можно считать функционально эквивалентным проводу, который он и заменяет в рамках решения этой задачи. Поиску функциональной эквивалентности предметов может способствовать их сходство или близость по целому ряду параметров. Такое сходство может быть представлено не только в визуальной, но и в вербальной модальности. Хотя сами авторы и не предлагают использовать данную особенность функциональной фиксированности для 
обращения с ней, эта модель, по нашему мнению, может быть использована в практике снижения функциональной фиксированности: в том случае, если необходимо найти некоторое применение «старым» инструментам, может быть полезно рассмотреть их в как можно большем количестве «измерений». Кроме этого, Глюксбергом (Glucksberg, 1962) были выделены два типа проявления функциональной фиксированности. Первый, описанный выше, заключается в неспособности воспринять фиксированный объект как релевантный данной задаче. Второй же проявляется в неспособности разделить между собой релевантные и нерелевантные функции объекта. Исходя из этого, для преодоления функциональной фиксированности он предлагает не просто вербально обозначать все элементы задачи, но и разделять в этих обозначениях их релевантные и нерелевантные (относительно текущей задачи) аспекты.

В работах П. Саугстада и К. Райхема (Saugstad, Raaheim, 1957, 1960) также высказывается предположение о том, что феномен функциональной фиксированности возникает из-за отсутствия у субъекта способности осознать релевантную задаче функцию целевого предмета. Для снижения уровня функциональной фиксированности авторы предлагают обращать внимание субъекта на те (зачастую многочисленные и, на первый взгляд, нерелевантные поставленной задаче) функции предметов, которые могут оказаться полезными для успешного решения.

Еще одним видом деятельности, способствующим снижению уровня функциональной фиксированности, может являться называние тех объектов, которые в меньшей степени способны реализовывать основную функцию целевого предмета (Yagolkovskiy, Medvedev, 2020). Так, например, если субъект перечисляет объекты, которые выполняют основную функцию фонарика (светить) хуже, чем этот фонарик, то при выполнении творческого задания с целевым объектом он/она будет в меньшей степени обращаться к этому свойству. Это способствует повышению оригинальности продуцируемых субъектом идей.

И. Мальтцман и др. (Maltzman et al., 1958), исходя из представления о креативности как способности реализовывать реакции, находящиеся на низких уровнях поведенческой иерархии, предлагают несколько способов повышения оригинальности ответов, которые можно использовать и для преодоления функциональной фиксированности. Так, первый заключается в постоянном варьировании условий задачи, чтобы побудить субъекта производить необычные реакции. Второй основывается на «провокации» - предъявлении нескольких необычных вариантов ответа, которые, согласно гипотезе авторов, в дальнейшем могут существенно облегчить возникновение других нестандартных решений.

Р. Арнон и С. Крейтлер (Arnon, Kreitler, 1984) высказывают предположение о том, что феномен функциональной фиксированности возникает в первую очередь из-за того, что ключевые элементы задачи рассматриваются человеком в крайне ограниченном пространстве значений, и предлагают для его преодоления использовать метод «meaning training», который основан на рас- 
ширении сферы значений целевого предмета. Авторы выделили 10 измерений значений:

- определение контекста (определение некоторой структуры или концепции, к которой принадлежит элемент задачи; например: нога - часть тела); ей);

- функция/предназначение (например: батарейка - обеспечение энерги-

- возможные действия (то, какие действия может реализовывать элемент; например: выключатель - может переключаться);

- диапазон включения (какие части в себя включает или может включать элемент; например: нож - рукоятка и лезвие);

- сенсорные качества (например: яблоко - круглое, зеленое;

- материал (например: свечка - воск);

- размер (например: микроб - очень маленький);

- вес и объем (например: бутылка - двести миллилитров);

- пространственные характеристики (могут быть описаны как в абсолютном, так и в относительном измерении; например: батарейка - в любом электрическом приборе, море - на юге);

- область применения (указание других элементов, к которым применяется или с которыми взаимодействует целевой элемент; например: отвертка гайки и болты, эмоции - люди и животные испытывают эмоции), сходство (например: море - похоже на небо).

B рамках «meaning training» человеку предлагается назвать хотя бы три значения по каждому из вышеперечисленных измерений, прежде чем начать выполнение задачи. Следует отметить, что в рамках исследования эффективности данной методики авторы смогли подтвердить лишь то, что она помогает решать поставленные задачи быстрее. При этом не было показано, что использование этого метода позволяет увеличить вероятность успешного решения творческой задачи.

Е. Хрисикоу (Chrysikou, 2006) уделяет особое внимание процессу категоризации объектов в процессе творческого мышления. Она выделяет два способа категоризации: таксономический (выделение стандартных родовых категорий: яблоко принадлежит к категории «фрукт», молоток - к категории «инструмент») и целеориентированный (goal-derived - выделение тех категорий объекта, которые релевантны конкретной ситуации). При этом целеориентированные категории можно разделить на устоявшиеся, которые уже были сформированы в более раннем опыте, и специальные, которые находятся в процессе формирования путем комбинирования таксономических или устоявшихся целеориентированных категорий. Автор предполагает, что именно способность формировать специальные категории является одной из наиболее значимых в условиях творческого процесса. В статье она приводит следующий пример: для того чтобы найти решение дункеровской задачи со свечой, субъекту необходимо уйти от таксономической категории коробки с кнопками - «коробка», и сформировать категорию «нечто, на что можно вертикально поставить свечу». В своем исследовании она демонстрирует, что обучение человека процессу «специальной», контекстуальной категоризации 
значительно повышает его способность преодолевать функциональную фиксированность и находить решения творческих задач.

Снижению функциональной фиксированности может способствовать и полное отвлечение от реализуемой деятельности. Дж.Г. Лу с соавт. (Lu et al., 2017) анализировали роль переключения между различными задачами в творческой деятельности субъекта. Было отмечено, что, несмотря на то, что, как показано в ряде исследований, частые переключения в выполняемой субъектом деятельности могут стать причиной ряда проблем (повышение вероятности совершения ошибки, замедление темпа деятельности, падение уровня обучаемости и запоминания, повышение уровня тревожности и пр.), они могут оказывать положительное влияние на эффективность решения творческих задач. Было показано, что любая деятельность, ведущая к отвлечению от целевой задачи (будь то переключение на другую деятельность, или просто перерыв, или даже борьба с возникающими помехами), способна снижать уровень «когнитивной фиксации», одним из выражений которой является и феномен функциональной фиксированности.

Схожая идея фигурировала и в работе Р.Е. Адамсона и Д.У. Тейлора (Adamson, Taylor, 1954). В рамках этого исследования проверялась гипотеза об ослаблении связи между предметом и его конкретной функцией в случае увеличения временно́го интервала между первичным использованием этого предмета и началом выполнения основной задачи. Оказалось, что функциональная фиксированность снижается с увеличением промежутка времени после первоначального использования предмета. И хотя сами авторы не говорили о возможности применения указанной особенности функциональной фиксированности в контексте обращения с ней, эта идея также может быть использована в практике снижения функциональной фиксированности: в том случае, если необходимо снизить уровень фиксированности субъекта на какой-либо функции объекта, перед предъявлением этому субъекту основной задачи следует сделать некоторую паузу в обращении с этим предметом.

Однако следует отметить, что сами Адамсон и Тейлор предполагали, что данный эффект не связан со временем как таковым, а скорее объясняется ретроактивным торможением функциональной фиксированности: активность, в которую был вовлечен субъект в период между первичным использованием предмета и началом выполнения основной задачи, мешала сохранению образовавшихся ассоциаций. Это могло позитивно сказаться на эффективности его/ее творческой деятельности.

Данное предположение во многом согласуется с теорией «забывания фиксации» (forgetting fixation theory), предложенной С. Смитом (Smith, 1994). В основе этой теории лежит представление о том, что функциональная фиксированность связана с неадекватно применяемым знанием об объекте в рамках конкретной ситуации. В том же случае, если когнитивные блоки, основанные на знании о функции этого объекта, становятся менее доступными и забываются, творческое решение можно будет находить более просто. Такой позитивный эффект может быть усилен выполнением различных задач на пере- 
ключение (например: переключением между категориями в задачах на генерацию идей - Smith et al., 2017).

Следует отметить, что сам факт возникновения функциональной фиксированности еще не означает, что процесс творческого мышления будет нарушен. В ряде случаев некоторый уровень фиксированности способен оказывать положительное влияние на творческий мыслительной процесс.

Так, Л. Дусинк и Л. Латур (Dusink, Latour, 1996) в своей работе предположили, что существует способ контролирования уровня функциональной фиксированности в рамках решения задач, который позволит, с одной стороны, использовать уже имеющиеся в опыте идеи, а с другой - видоизменять их и делать более универсальными. Для этого они предлагают следующие рекомендации, которые могут повысить эффективность творческой деятельности субъекта:

- оспаривание предположений: при принятии любого решения необходимо разъяснять, почему принимается именно такое решение, откуда оно берется и на что опирается;

• осознание импликаций (make implications clear): перед реализацией конкретного решения следует четко понимать, какие именно факторы влияют на его принятие;

- эксплицирование аналогий: при использовании аналогий крайне важно делать их явными, прояснять их;

- признание важности прошлого опыта: все три предыдущих принципа должны реализовываться в рамках осознания своего опыта как чрезвычайно важного ресурса для успешного решения творческой задачи.

И. Соломон (Solomon, 1994) также продемонстрировала, что в некоторых случаях использование прошлого опыта ведет не к затруднениям в решениях задач, а, наоборот, способствует продуцированию новых идей. Она заметила, что если субъект использует примеры из прошлого, опираясь на осознание их глубинной структуры, а не на поверхностные признаки, то это может привести к росту эффективности решения творческой задачи. Возможное позитивное влияние прошлого опыта решения задачи на продуцирование идей в условиях последующих попыток ее решения обсуждалось также в рамках «гипотезы оппортунистической ассимиляции», предложенной Сейферт с соавт. (Seifert et al., 1995). Согласно этой модели, безуспешные попытки решения задачи оставляют след в памяти субъекта. Этот след может позволить более эффективно продолжить решение задачи при появлении новых релевантных стимулов.

Большинство перечисленных способов ослабления функциональной фиксированности имеют одно важное сходство: они опираются на представление об этом феномене как о неосознаваемом процессе, сужающем область восприятия объекта и препятствующем рассмотрению всех сторон и связей объекта. Это ограничивает проявление творческого потенциала субъекта во взаимодействии с ним. Образ объекта становится более бедным и простым, содержащим меньшее количество ассоциативных элементов, которое не может предоставить достаточного материала для высокой дивергентности мышления 
при решении задач или генерировании идей, связанных с этим объектом. Роль уровня сложности воспринимаемого объекта в дивергентном мышлении отмечалась в целом ряде работ (например: Jamieson, 1974; Kharkhurin, Yagolkovskiy, 2019). Поэтому особую важность для снижения выраженности функциональной фиксированности и повышения эффективности творческого мышления приобретает «расширение поля» восприятия объекта или проблемной ситуации, позволяющее сформировать их более полный и сложный образ. Эффективным способом ослабления функциональной фиксированности может стать расширение ассоциативного базиса: привлечение к выполнению творческого задания сразу нескольких человек, уделение более пристального внимания конкретным дополнительным свойствам целевого предмета, разложение его образа на составляющие, переключение на другие задачи и пр. Все эти меры способствуют «обогащению» ассоциативного пространства и повышению эффективности мыслительного процесса через увеличение числа направлений, в которых оно может осуществляться. Это может приводить к повышению уровня дивергентности творческого мышления и выводу его за пределы «линейной» траектории, ограничивающей реализацию творческого потенциала человека (Acar, Runco, 2019).

В представленной статье были описаны и кратко проанализированы основные методы снижения и нейтрализации функциональной фиксированности. Некоторые из них основываются на общих рекомендациях к реализации творческого процесса, другие ориентированы на предъявление субъекту конкретных инструкций. Общей чертой большинства проанализированных в этой работе способов преодоления функциональной фиксированности является их акцент на обогащении и расширении различными способами ассоциативного пространства, служащего источником идей и нестандартных решений в рамках творческой деятельности субъекта.

Следует отметить, что в настоящее время в научной литературе по этой тематике проявляется тенденция к выделению и анализу отдельных компонентов и аспектов функциональной фиксированности. Это может привести к возникновению в ближайшем будущем более таргетированных и эффективных методов ее ослабления, что позволит повысить продуктивность творческой деятельности субъекта.

\section{Литература}

Медведев, Б. П., Яголковский, С. Р. (2020). Функциональная фиксированность и ее роль в снижении продуктивности творческого мышления. Психология. Журнал Высшей школь экономи$\kappa и, 17(3), 414-427$.

Ссылки на зарубежные источники см. в разделе References после англоязычного блока. 
Яголковский Сергей Ростиславович - старший научный сотрудник, Национальный исследовательский университет «Высшая школа экономики», кандидат психологических наук, доцент. Сфера научных интересов: креативность, групповое творчество, методы стимуляции креативности.

Контакты: syagolkovsky@hse.ru

Медведев Богдан Павлович - аспирант, Национальный исследовательский университет «Высшая школа экономики».

Сфера научных интересов: креативность, персонология, психология сознания.

Контакты: bmedvedev@hse.ru 


\title{
Psychological Methods to Loosen Functional Fixedness
}

\author{
S.R. Yagolkovskiy ${ }^{\mathrm{a}}$, B.P. Medvedev ${ }^{\mathrm{a}}$ \\ ${ }^{a}$ HSE University, 20 Myasnitskaya Str., Moscow, 101000, Russian Federation
}

\begin{abstract}
This is the second part of research on functional fixedness, a phenomenon of inhibition of creative thinking. Numerous methods to loosen functional fixedness are analyzed. The core principle of these methods is the enrichment of associative space serving as a source for creative ideas and solutions. Some ways to loosen functional fixedness are based on theories and methods presented in scientific literature (e.g., forgetting fixation theory (Smith, 1994), opportunistic assimilation hypothesis (Seifert et al., 1994), etc.). Methods aimed at reducing functional fixedness differ from each other by the degree of generalization. Some of them are based on general recommendation of how to improve creative thinking, other methods are more specific providing detailed instruction what to do. Different parameters of these instructions and ways of their presentation are analyzed. We also examine the role of categorization, past experience, and switching to another activity as factors affecting functional fixedness. Additionally, other approaches are considered, which based on the assumption that this phenomenon is not an unambiguous antagonist of creative thinking, but can help to increase its effectiveness. It takes place if a subject is aware of the influence of past experience on creative thinking and deeply understands the structure of the problem to be solved. In research on functional fixedness, there is a tendency to identify and examine its specific components. This can lead to the development of new targeted methods to loosen functional fixedness.
\end{abstract}

Keywords: functional fixedness, creative thinking, problem-solving set, divergent thinking.

\section{References}

Acar, S., \& Runco, M. A. (2019). Divergent thinking: New methods, recent research, and extended theory. Psychology of Aesthetics, Creativity, and the Arts, 13(2), 153-158. https://doi.org/10.1037/aca0000231

Adamson, R. E. (1952). Functional fixedness as related to problem solving: a repetition of three experiments. Journal of Experimental Psychology, 44(4), 288-291. https://doi.org/10.1037/h0062487

Adamson, R. E., \& Taylor, D. W. (1954). Functional fixedness as related to elapsed time and to set. Journal of Experimental Psychology, 47(2), 122-126. https://doi.org/10.1037/h0057297

Arnon, R., \& Kreitler, S. (1984). Effects of meaning training on overcoming functional fixedness. Current Psychology, 3(4), 11-24. https://doi.org/10.1007/BF02686553

Carnevale, P. J., \& Probst, T. M. (1998). Social values and social conflict in creative problem solving and categorization. Journal of Personality and Social Psychology, 74(5), 13001309. https://doi.org/10.1037/0022-3514.74.5.1300

The reported study was funded by RFBR, project N 19-113-50017. 
Chi, M. T. H., Feltovich, P. J., \& Glaser, R. (1981). Categorization and representation of physics problems by experts and novices. Cognitive Science, 5(2), 121-152. https://doi.org/10.1207/s15516709cog0502_2

Chrysikou, E. G. (2006). When shoes become hammers: Goal-derived categorization training enhances problem-solving performance. Journal of Experimental Psychology: Learning Memory and Cognition, 32(4), 935-942. https://doi.org/10.1037/0278-7393.32.4.935

Chrysikou, E. G., Motyka, K., Nigro, C., Yang, S.-I, \& Thompson-Schill, S. L. (2016). Functional fixedness in creative thinking tasks depends on stimulus modality. Psychology of Aesthetics, Creativity, and the Arts, 10(4), 425-435. https://doi.org/10.1037/aca0000050

Chrysikou, E. G., \& Weisberg, R. W. (2005). Following the wrong footsteps: fixation effects of pictorial examples in a design problem-solving task. Journal of Experimental Psychology: Learning, Memory, and Cognition, 31(5), 1134-1148. https://doi.org/10.1037/0278-7393.31.5.1134

Coskun, H., Paulus, P., Brown, V., \& Sherwood, J. (2000). Cognitive stimulation and problem presentation in idea generation groups. Group Dynamics: Theory, Research, and Practice, 4(4), 307-329. https://doi.org/10.1037//1089-2699.4.4.307

Duncker, K. (1945). On problem-solving. Psychological Monographs, 58(5), 1-113. https://doi.org/10.1037/h0093599

Dusink, L., \& Latour, L. (1996). Controlling functional fixedness: the essence of successful reuse. Knowledge-Based Systems, 9(2), 137-143. https://doi.org/10.1016/0950-7051(95)01025-4

Frank, M. C., \& Ramscar, M. (2003). How do presentation and context influence representation for functional fixedness tasks? Proceedings of the Annual Meeting of the Cognitive Science Society, 25, 1345. https://escholarship.org/uc/item/1tq4b850

Glaser, W. R., \& Glaser, M. O. (1989). Context effects in stroop-like word and picture processing. Journal of Experimental Psychology: General, 118(1), 13-42. https://doi.org/10.1037/00963445.118.1.13

Glucksberg, S. (1962). The influence of strength of drive on functional fixedness and perceptual recognition. Journal of Experimental Psychology, 63(1), 36-41. https://doi.org/10.1037/h0044683

Glucksberg, S., \& Danks, J. H. (1967). Functional fixedness: Stimulus equivalence mediated by semantic-acoustic similarity. Journal of Experimental Psychology, 74(3), 400405. https://doi.org/10.1037/h0024724

Glucksberg, S., \& Weisberg, R. W. (1966). Verbal behavior and problem solving: Some effects of labeling in a functional fixedness problem. Journal of Experimental Psychology, 71(5), 659-664. https://doi.org/10.1037/h0023118

Jamieson, S. E. (1974). An examination of preference for complexity and its relation to creativity (Doctoral dissertation, Durham University).

Kearsley, G. P. (1975). Problem-solving set and functional fixedness: A contextual dependency hypothesis. Canadian Psychological Review/Psychologie canadienne, 16(4), 261-268. https://doi.org/10.1037/h0081813

Kharkhurin, A. V., \& Yagolkovskiy, S. R. (2019). Preference for complexity and asymmetry contributes to elaboration in divergent thinking. Creativity Research Journal, 31(3), 342-348. https://doi.org/10.1080/10400419.2019.1641687

LeFevre, J. A., \& Dixon, P. (1986). Do written instructions need examples? Cognition and Instruction, 3(1), 1-30. https://doi.org/10.1207/s1532690xci0301_1 
Lu, J. G., Akinola, M., \& Mason, M. F. (2017). "Switching On” creativity: Task switching can increase creativity by reducing cognitive fixation. Organizational Behavior and Human Decision Processes, 139, 63-75. https://doi.org/10.1016/j.obhdp.2017.01.005

Maltzman, I., Brooks, L. O., Bogartz, W., \& Summers, S. S. (1958). The facilitation of problem solving by prior exposure to uncommon responses. Journal of Experimental Psychology, 56(5), 399-406. https://doi.org/10.1037/h0046758

McCaffrey, A. J. (2012). The obscure features hypothesis for innovation: One key to improving performance in insight problems (Doctoral dissertation, University of Massachusetts Amherst).

Medvedev, B., \& Yagolkovskiy, S. (2020). Functional fixedness and Its role in reducing productivity of creative thinking. Psychology. Journal of Higher School of Economics, 17(3), 414-427. https://doi.org/10.17323/1813-8918-2020-3-414-427 (in Russian)

Munoz-Rubke, F., Olson, D., Will, R., \& James, K. H. (2018). Functional fixedness in tool use: Learning modality, limitations and individual differences. Acta Psychologica, 190, 11-26. https://doi.org/10.1016/j.actpsy.2018.06.006

Ohlsson, S. (1992). Information processing explanations of insight and related phenomena. In M. T. Keane \& K. J. Gilhooly (Eds.), Advances in the psychology of thinking (pp. 1-44). London: Harvester Wheatsheaf.

Pirolli, P. L., \& Anderson, J. R. (1985). The role of practice in fact retrieval. Journal of Experimental Psychology: Learning, Memory, and Cognition, 11(1), 136-153. https://doi.org/10.1037/02787393.11.1.136

Saffran, E. M., Coslett, H. B., Martin, N., \& Boronat, C. (2003). Access to knowledge from pictures but not words in a patient with progressive fluent aphasia. Language and Cognitive Processes, 18(5-6), 725-757. https://doi.org/10.1080/01690960344000107

Saugstad, P., \& Raaheim, K. (1957). Problem solving and availability of functions. Acta Psychologica, 13(1), 263-278. https://doi.org/10.1016/0001-6918(57)90026-4

Saugstad, P., \& Raaheim, K. (1960). Problem solving, past experience and availability of functions. British Journal of Psychology, 51(2), 97-104. https://doi.org/10.1111/j.20448295.1960.tb00730.x

Seifert, C. M., Meyer, D. E., Davidson, N., Patalano, A. L., \& Yaniv, I. (1995). Demystification of cognitive insight: Opportunistic assimilation and the prepared-mind perspective. In R. J. Sternberg \& J. E. Davidson (Eds.), The nature of insight (pp. 65-124). Cambridge, MA: MIT Press.

Smith, S. M. (1994). Getting into and out of mental ruts: a theory of fixation, incubation, and insight. In R. J. Sternberg \& J. Davidson (Eds.), The nature of insight (pp. 229-251). Cambridge, MA: MIT Press.

Smith, S. M., Gerkens, D. R., \& Angello, G. (2017). Alternating incubation effects in the generation of category exemplars. Journal of Creative Behavior, 51(2), 95-106. https://doi.org/10.1002/jocb.88

Solomon, I. (1994). Analogical transfer and "functional fixedness" in the science classroom. The Journal of Educational Research, 87(6), 371-377. https://doi.org/10.1080/00220671.1994.9941268

Sternberg, R. J. (1996). Cognitive psychology. Orlando, FL: Harcourt-Brace.

Voiskounsky, A. E., Yermolova, T., Yagolkovskiy, S. R., \& Chromova, V. M. (2017). Creativity in online gaming: individual and dyadic performance in Minecraft. Psychology in Russia: State of the Art, 10(4), 144-161. https://doi.org/10.11621/pir.2017.0413

Yagolkovskiy, S. R., \& Medvedev, B. P. (2020). Enhancement of creativity: semantic priming through naming objects loosens functional fixedness within idea generation. The Journal of Creative Behavior, 54(4), 1013-1020. https://doi.org/10.1002/jocb.422 
Sergey R. Yagolkovskiy - Senior Research Fellow, HSE University, PhD in Psychology, Associate Professor.

Research Area: creativity, group creativity, methods of stimulating creativity.

E-mail: syagolkovsky@hse.ru

Bogdan P. Medvedev - PhD student, HSE University.

Research Area: creativity, personology, psychology of consciousness.

E-mail: bmedvedev@hse.ru 